Case report

\title{
Different responses to two types of 5-fluorouracil prodrugs in combination with interferon-alpha in pulmonary metastases of renal cell carcinoma: a case report Tatsuo Morita*, Kazuhiko Nakano and Masayuki Yuzawa
}

Address: Department of Urology, Jichi Medical University, Yakushiji 3311-1, Shimotsuke-city, Tochigi 3290498, Japan

Email: TM* - moritatu@jichi.ac.jp; KN - nknkzhk@jichi.ac.jp; MY - m.yuzawa@jichi.ac.jp

* Corresponding author

Published: 26 May 2009

Received: 14 March 2009

Cases Journal 2009, 2:6567 doi: 10.1186/1757-1626-2-6567

Accepted: 30 April 2009

This article is available from: http://casesjournal.com/casesjournal/article/view/6567

(c) 2009 Morita et al; licensee Cases Network Ltd.

This is an Open Access article distributed under the terms of the Creative Commons Attribution License (http://creativecommons.org/licenses/by/3.0), which permits unrestricted use, distribution, and reproduction in any medium, provided the original work is properly cited.

\begin{abstract}
A 66-year-old Japanese man with pulmonary metastases of renal cell carcinoma found 8 months after radical nephrectomy was treated with interferon-alpha and tegafur-uracil. Since it failed to achieve tumor responses resulting in progression, he was given interferon-alpha and capecitabine. After 2 courses of combination therapy with IFN-alpha and capecitabine, significant tumor responses were obtained; two out of four pulmonary metastatic sites disappeared completely, one site showed over $50 \%$ decrease in size, and the remaining one site did no change in size. The regimen was well tolerated and toxicity observed was World Health Organization grade I anorexia. His disease status was maintained as stable disease by the repeated treatment with interferon-alpha and capecitabine for 17 months after tumor responses were obtained. However, tumor progression was observed thereafter. He is at present under treatment with sorafenib. This is the first case report of metastatic renal cell carcinoma, which showed different responses to two types of 5-fluorouracil prodrugs in combination with interferon-alpha, suggesting the biochemical modulation of capecitabine by interferon-alpha as a possible mechanism underlying the antitumor effect of the combination of interferon-alpha and capecitabine at the clinical setting. Present case also suggests that a combination of tumor-selective capecitabine with interferon-alpha is a potentially useful therapeutic option in metastatic renal cell carcinoma.
\end{abstract}

\section{Introduction}

Current standard therapy against metastatic renal cell carcinoma (RCC) is moving from the cytokine-based therapy to the inhibition of angiogenesis with targeted agents. Since the latter is indeed promising but is not curative, we need to explore the further treatment regimens, which would benefit RCC patients. Cytokines, such as interleukin-2 (IL-2) and/or interferon (IFN) produce responses in $10-15 \%$ of patients, with occasional complete responses reported [1]. On the other hand, tumor response rates of $13 \%$ to $43 \%$ in the treatment of metastatic RCC have been reported by immunochemotherapy consisting of IFN-alpha and fluoropyrimidines with or without IL-2 [2-4]. Capecitabine is an orally administered, 
tumor-selective fluoropyrimidine that is converted to 5-fluorouracil (5FU) by three enzymes: carboxylesterase mainly located in the liver, cytidine deaminase in the liver and tumors, and thymidine phosphorylase (TP) in tumors [5]. In clinical trials in metastatic RCC, combination of IFN-alpha and tumor-selective capecitabine has a slightly superior overall response rate compared with capecitabine monotherapy: $12-24 \%[6,7]$ versus $4.8-8.7 \%$, respectively $[8,9]$. Herein, we report a case of pulmonary metastases of RCC, which were resistant to the treatment with IFN-alpha and tegafur-uracil (UFT) but were sensitive to the treatment with IFN-alpha and capecitabine.

\section{Case presentation}

A 66-year-old Japanese man with right RCC (cT2NOM0) which was detected incidentally by ultrasound of health checkup underwent radical nephrectomy on October 2005 (Figure 1). Pathological examination revealed clear cell carcinoma with an alveolar arrangement (pT3N0). After the operation, he was followed up periodically without any further treatments. On June 2006, systemic examination demonstrated four coin lesions in bilateral lungs consistent with multiple pulmonary metastases of RCC without any other metastatic sites as shown in Figure 2 (A, D, G, J). He received combination therapy with IL-2, IFN-alpha (Sumiferon ${ }^{\mathrm{TM}}$ ), and UFT as reported previously [10]. It was discontinued immediately because of the skin toxicity due to IL-2. Then, he was given UFT at a daily dose of $300 \mathrm{mg}$ three times a day and natural IFN-alpha (Sumiferon ${ }^{\mathrm{TM}}$ ) 3 million $\mathrm{U}$ intramuscularly five times a

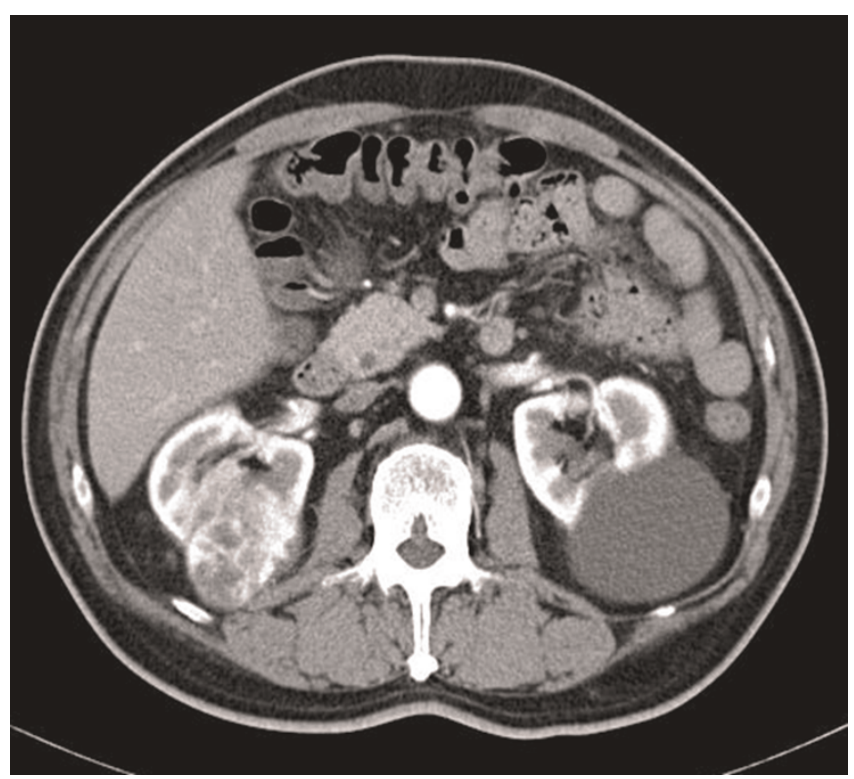

Figure I. Abdominal CT before radical nephrectomy. Abdominal CT showed right hypervascular renal mass compatible with RCC. week in a course of 3 weeks on/1 week off as the first line treatment. However, chest computed tomography (CT) showed progression of the disease (PD) after two courses of the treatment. We switched 3 million U of IFN-alpha (Sumiferon ${ }^{\mathrm{TM}}$ ) to 5 million $\mathrm{U}$ of natural IFN-alpha $\left(\mathrm{OIF}^{\mathrm{TM}}\right)$ in combination with UFT based on a case report that metastatic RCC which did not respond to natural IFNalpha (Sumiferon ${ }^{\mathrm{TM}}$ ) did respond to another type of natural IFN-alpha $\left(\mathrm{OIF}^{\mathrm{TM}}\right)$ [11]. However, two courses of natural IFN-alpha $\left(\mathrm{OIF}^{\mathrm{TM}}\right)$ in combination with UFT as the second line treatment failed to achieve tumor responses and PD was observed again as shown in Figure 2 (B, E, H, K). Combination therapy with IFN-alpha $\left(\mathrm{OIF}^{\mathrm{TM}}\right)$ and UFT was discontinued. On May 2007, he was given capecitabine at a daily dose of $2400 \mathrm{mg}$ twice a day and natural IFN-alpha $\left(\mathrm{OIF}^{\mathrm{TM}}\right) 5$ million $\mathrm{U}$

$\# 1$

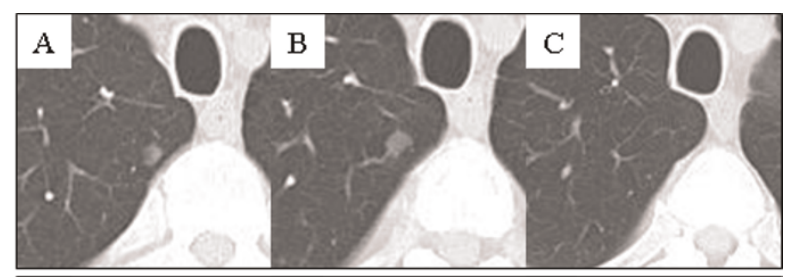

$\# 2$

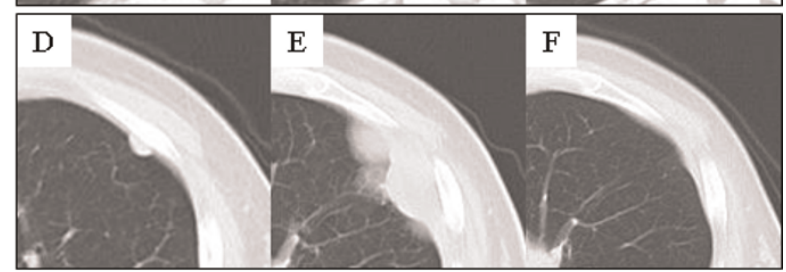

\#3

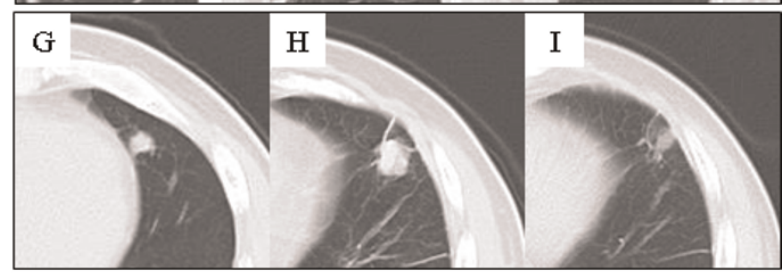

\#4

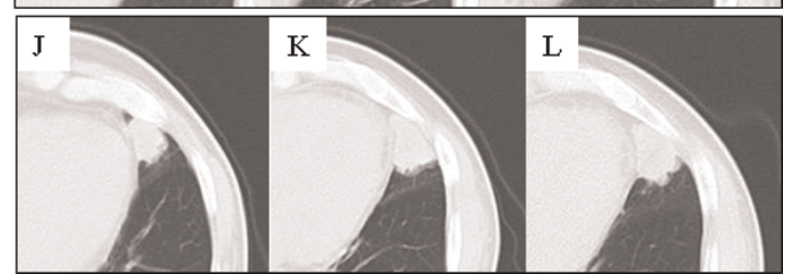

Figure 2. Chest CT before and after the combination therapy with IFN-alpha and capecitabine. Pulmonary metastases were found 8 months after radical nephrectomy $(\mathbf{A}, \mathbf{D}, \mathbf{G}, \mathbf{J})$. Combination therapy with IFN-alpha and UFT failed to achieve tumor responses and PD was observed $(\mathbf{B}, \mathbf{E}, \mathbf{H}, \mathbf{K})$. Two (\#I, \#2) out of four pulmonary metastatic sites disappeared completely, one site (\#3) showed over $50 \%$ decrease in size, and the remaining one site (\#4) did no change in size after 2 courses of the combination therapy with IFN-alpha and capecitabine (C,F,I,L). 
intramuscularly three times a week in a course of 2 weeks on/ 1 week off as the third line treatment. After 2 courses of the combination therapy with IFN-alpha and capecitabine, significant tumor responses were obtained; two out of four pulmonary metastatic sites disappeared completely, one site showed over $50 \%$ decrease in size, and the remaining one site did no change in size as shown in Figure 2 (C, F, I, L). This regimen was well tolerated and toxicity observed was World Health Organization (WHO) grade 1 anorexia without hand-foot syndrome. His disease status was maintained as stable disease by the repeated treatment with IFN-alpha and capecitabine for 17 months after tumor responses were obtained. However, tumor progression was observed thereafter. He is at present under treatment with sorafenib.

\section{Discussion}

Present case is unique in terms of different responses to two types of 5FU prodrugs. Namely, pulmonary metastases of RCC resistant to the treatment with IFN-alpha and UFT were sensitive to the treatment with IFN-alpha and capecitabine. Following possible explanations may account for the different responses to two types of 5FU prodrugs in combination with IFN-alpha Firstly, TP expression in tumor tissues might be involved in the causes of different responses to two types of 5FU prodrugs. $\mathrm{TP}$ is a rate-limiting enzyme for capecitabine and involved much in the anabolic pathway of capecitabine rather than UFT. Ishikawa et al. [12] showed that TP expression level in tumor tissues correlated well with the efficacy of capecitabine but not UFT in human cancer xenograft model, suggesting that TP expression level would be a predictive factor for capecitabine. TP expression in RCC is also independent prognostic factor for RCC patients and varies among them [13-15]. Thus, we examined and evaluated TP expression in the resected kidney of the present case by immunohistochemistry as reported previously [14], and confirmed that it had medium to high TP expression level (data not shown). Although TP expression level in primary site is not always the same as that in metastatic sites, immunohistochemistry suggests that RCC in the present case might be sensitive to capecitabine in terms of TP expression resulting in the significant tumor responses to capecitabine. Secondly, biochemical modulation in which a modulator combined with anti-tumor drugs changes pharmacokinetics and results in increased efficacy and/or reduction of toxicity would be expected in capecitabine when administered in combination with IFNalpha. Transfection studies [16] showed direct evidence that enhancement of TP expression by transfection of TP cDNA makes RCC cell line more susceptible to capecitabine not only in vitro but also in vivo, suggesting that enhancement of TP expression in tumor tissues by modulators such as IFN-alpha would increase capecitabine efficacy. We examined modulatory effect of IFN- alpha on 5FU and 5'-deoxy-5-fluorouridine (5'DFUR) which is the metabolite in the anabolic pathway of capecitabine with a particular focus on TP expression in human RCC cell lines [17], and showed that IFN-alpha upregulates TP expression and modulates fluoropyrimidine anabolism resulting in enhancement of the sensitivity to 5FU [17] and 5'DFUR [18] in RCC cell lines. Furthermore, extent of enhancement of the sensitivity to 5'DFUR by IFN-alpha was higher than that in 5FU [18]. Similar results were reported using human colon cancer cell line COLO 201 [19]. Taken together, we believe that biochemical modulation of capecitabine by IFN-alpha via TP might contribute to the significant tumor responses in the present case. Thirdly, the dose of UFT used in the present case might be insufficient to achieve the tumor responses. Although optimal dose of UFT in Japan ranges $300 \mathrm{mg}$ to $600 \mathrm{mg}$, we employed $300 \mathrm{mg}$ UFT because we experienced severe side effect of leukoencephalopathy-like symptoms in metastatic RCC patient in whom complete response (CR) was obtained by the combination of $600 \mathrm{mg}$ UFT and IFN-alpha as reported previously [20]. Akaza et al. reported that combination of 300 or $600 \mathrm{mg}$ UFT and IFN-alpha showed 3 CR cases and 2 partial response (PR) cases in 25 cases with metastatic RCC with a overall response rate of $20 \%$ [21]. Therefore, it cannot be excluded that $300 \mathrm{mg}$ UFT used in the present case was insufficient to achieve the tumor responses.

\section{Conclusion}

To our knowledge, this is the first case report of metastatic RCC which showed different responses to two types of $5 F U$ prodrugs in combination with IFN-alpha, suggesting the biochemical modulation of capecitabine by IFN-alpha as a possible mechanism underlying the antitumor effect of the combination of IFN-alpha and capecitabine at the clinical setting. Present case also suggests that a combination of tumor-selective capecitabine with IFN-alpha is a potentially useful therapeutic option in metastatic RCC.

\section{List of abbreviations}

RCC, Renal cell carcinoma; IL-2, interleukin-2; IFN, Interferon; 5FU, 5-fluorouracil; $\mathrm{TP}$, Thymidine phosphorylase; UFT, Tegafur-uracil; CT, Computed tomography; PD, Progression of the disease; WHO, World Health Organization; 5'DFUR, 5'-deoxy-5-fluorouridine.

\section{Consent}

Written informed consent was obtained from the patient for publication of this case report and any accompanying images. A copy of the written consent is available for review by the Editor-in-Chief of this journal.

\section{Competing interests}

The authors declare that they have no competing interests. 


\section{Author's contributions}

TM designed the treatment protocol and drafted the manuscript. KN evaluated the tumor responses and was involved in postoperative follow-up. MY was involved in critically revising the manuscript. All authors read and approved the final manuscript.

\section{References}

I. Bukowski RM, Novick AC: Clinical practice guidelines: renal cell carcinoma. Cleve Clin J Med 1997, 64: I-44.

2. Elias L, Blumenstein BA, Kish J, Flanigan RC, Wade JL, Lowe BA, Goodwin JW, Crawford ED: A phase II trial of interferon-alpha and 5 -fluorouracil in patients with advanced renal cell carcinoma. A Southwest Oncology Group Study. Cancer 1996, 78:1085-1088.

3. Gebrosky NP, Koukol S, Nseyo UO, Carpenter C, Lamm DL: Treatment of renal cell carcinoma with 5-fluorouracil and alpha-interferon. Urology 1997, 50:863-867.

4. Atzpodien J, Kirchner $\mathrm{H}$, Illiger HJ, Metzner B, Ukena D, Schott $\mathrm{H}$, Funke PJ, Gramatzki M, Jürgenson S, Wandert T, Patzelt T, Reitz M: IL-2 in combination with IFN-alpha and 5-FU versus tamoxifen in metastatic renal cell carcinoma: long-term results of a controlled randomized clinical trial. $\mathrm{Br} J$ Cancer 200I, 85: II30-II36.

5. Morita T, Matsuzaki A, Suzuki K, Tokue A: Role of thymidine phosphorylase in biomodulation of fluoropyrimidines. Curr Pharm Biotech 200I, 2:257-267.

6. Padrik P, Leppik K, Arak A: Combination therapy with capecitabine and interferon alpha-2A in patients with advanced renal cell carcinoma: a phase II study. Urol Oncol 2004, 22:387-392.

7. Segota E, Mekhail T, Olencki T, Hutson TE, Dreicer R, Wacker B, Osterwalder B, Elson P, Zhou M, Bukowski RM: Phase II trial of capecitabine and rHu-interferon-alpha-2a in patients with metastatic renal cell carcinoma, limited efficacy, and moderate toxicity. Urol Oncol 2007, 25:46-52.

8. Wenzel C, Locker G], Bartsch R, Pluschnig U, Mader R, Hussian D, Kramer G, Marberger M, Lintner C, Rauchenwald M, Zielinski CC, Steger GG: Capecitabine monotherapy and in combination with immunotherapy in the treatment of metastatic renal cell carcinoma. Anticancer Drugs 2003, 14:779-784.

9. Petrioli R, Paolelli L, Francini E, Marsili S, Pascucci A, Sciandivasci A, de Rubertis G, Barbanti G, Manganelli A, Salvestrini F, Francini G: Capecitabine as third-line treatment in patients with metastatic renal cell carcinoma after failing immunotherapy. Anticancer Drugs 2007, I 8:817-820.

10. Kobayashi M, Ikeda $H$, Nukui A, Suzuki K, Sugaya $Y$, Yuzawa M, Morita T: Clinical outcome and prognostic survival factors in patients with advanced renal cell carcinoma treated with very low-dose interleukin-2, interferon-alpha, and tegafururacil: a single-institution experience. Int J Clin Oncol 2008, 13:257-262.

II. Oya M, Asakura H, Mizuno R, Marumo K, Murai M: Repeated regression of pulmonary metastases from renal cell carcinoma after treatment using different interferon-alpha preparations. Biomed Res 2005, 26:135-137.

12. Ishikawa T, Sekiguchi F, Fukase Y, Sawada N, Ishitsuka H: Positive correlation between the efficacy of capecitabine and doxifluridine and the ratio of thymidine phosphorylase to dihydropyrimidine dehydrogenase activities in tumors in human cancer xenografts. Cancer Res 1998, 58:685-690.

13. Imazano $Y$, Takebayashi $Y$, Nishiyama $K$, Akiba $S$, Miyadera $K$, Yamada Y, Akiyama S, Ohi Y: Correlation between thymidine phosphorylase expression and prognosis in human renal cell carcinoma. I Clin Oncol 1997, 15:2570-2578.

14. Suzuki K, Morita T, Hashimoto S, Tokue A: Thymidine phosphorylase/platelet-derived endothelial cell growth factor (PDECGF) associated with prognosis in renal cell carcinoma. Urol Res 200I, 29:7-12.

15. Morita T, Matsuzaki A, Tokue A: Quantitative analysis of thymidine phosphporylase and dihydropyrimidine dehydrogenase in renal cell carcinoma. Oncology 2003, 65:|25-13|.
16. Morita T, Matsuzaki A, Tokue A: Enhancement of sensitivity to capecitabine in human renal carcinoma cells transfected with thymidine phosphporylase cDNA. Int J Cancer 200I, 92:45I-456.

17. Morita T, Tokue A: Biomodulation of $\mathbf{5}$-fluorouracil by interferon-alpha in human renal carcinoma cells: relationship to the expression of thymidine phosphorylase. Cancer Chemother Pharmacol 1999, 44:91-96.

18. Morita T: Biochemical modulation of fluoropyrimidines via thymidine phosphorylase. Jpn / Clin Urol 2007, 61:187-197.

19. Eda H, Fujimoto K, Watanabe S, Ura M, Hino A, Tanaka Y, Wada K, Ishitsuka $\mathrm{H}$ : Cytokines induce thymidine phosphorylase expression in tumor cells and make them more susceptible to 5'-deoxy-5-fluoropyrimidine. Cancer Chemother Pharmacol 1993, 32:333-338.

20. Suzuki K, Nukui A, Kobayashi M, Sugaya Y, Muraishi O, Morita T, Tokue A: A case of pulmonary metastasis from renal cell carcinoma with complete response to interferon-alpha and tegafur/uracil (UFT) but possibly UFT-induced liver dysfunction and leukoencephalopathy-like symptoms. Hinyokika Kiyo $1999,45: 621-624$

2I. Akaza H, Kameyama S, Kanemura M, Kitajima K, Homma $Y$, Kawamura T, Okada K, Murahashi I, Kawabe K, Isurugi K: Treatment of renal cell carcinoma with human lymphoblastoid interferon alpha and UFT in combination. A prospective multicenter trial. Jpn J Urol 1991, 82:1053-1058.

\section{Do you have a case to share?}

\author{
Submit your case report today \\ - Rapid peer review \\ - Fast publication \\ - PubMed indexing \\ - Inclusion in Cases Database
}

\section{Any patient, any case, can teach us something}

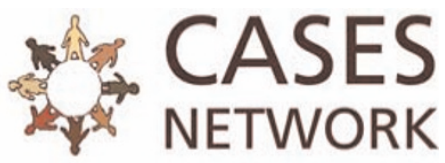

www.casesnetwork.com 